\title{
Tribulations d'un médecin coopérant sous les tropiques
}

Jean Martin

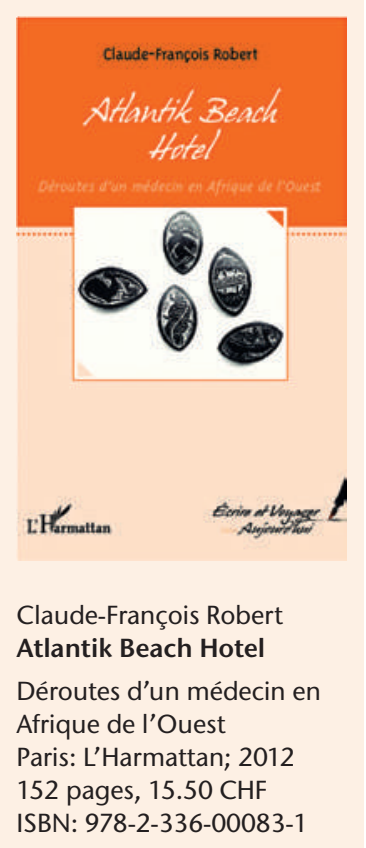

jean.martin[at]saez.ch
Le Dr Claude-François Robert est le médecin cantonal de Neuchâtel depuis 2007, après avoir travaillé en médecine interne puis principalement dans le domaine de la santé publique. Il a réalisé des missions en Afrique dans divers cadres, pour la coopération technique suisse et des organisations non gouvernementales, ainsi que pour un programme de collaboration entre l'Université de Genève et celle de Yaoundé. Dans un ouvrage qui vient de paraître il rassemble des tranches de vie en Afrique de l'Ouest, allant de l'engagement dans des régions en guerre à des projets de développement et des travaux de recherche.

Le personnage principal est dit «le toubib». Avertissement au début du livre: «C'est un être composite de plusieurs personnages qui se reconnaîtront éventuellement». Chapitres racontant chacun une histoire vécue (par l'auteur ou d'autres qui ont partagé avec lui leur expérience), une implication pourrait-on dire, en Afrique dans le domaine de la santé. Les premiers traitent de régions troublées ou en guerre, avec leur cortège de famine et aide alimentaire, de déplacements de populations, voire de massacres. Les protagonistes se retrouvent là où on vient échanger, décompresser, parlant des évènements du jour ou de politique locale ou internationale. Ainsi le bar «Dolce Vita» dans une capitale dont je me suis demandé, pour y avoir vécu deux ans une bonne décennie plus tôt, si c'était Yaoundé (ai ressenti comme des «vibrations» connues). Puis viennent des textes où c'est «Chez Otto» qui est de lieu de rencontres, de récits, le débat - la bière aidant.

Moments multiples et divers de la vie des coopérants. Ceux qui tiennent le coup, ceux qui noient les difficultés dans l'alcool (pas rare dans ces conditions de dépaysement et de surprises) et dégringolent, parfois de façon irréversible; Sarah qui fait un burnout perdurant au retour; Kim qui déprime, effet secondaire de l'antipaludique Lariam, dont le toubib retrouve le nom des années plus tard dans un magazine, devenue négociatrice commerciale pour le gouvernement américain. Les collaborateurs et collègues africains, personnel de santé, chauffeurs, piroguiers, villageois, fonctionnaires, chef coutumiers; ombre de la magie ici et là. Des notations sur les bureaux centraux des organismes de secours ou de coopération (qu'on dénomme parfois le Saint-Siège). Une description de ce que peut être le passage des motards et de la caravane du Paris-Dakar... Commentaires sur la poussée de la Chine en Afrique - phénomène majeur au plan géopolitique. Etudes épidémiologiques sur la bilharziose auxquelles le toubib participe autour d'un lac artificiel au Nord Cameroun.
Difficultés transculturelles: «Si tu n'es pas compris, au lieu de trouver que ton interlocuteur est un imbécile, il faut, toi, l'écouter. Quand tu le comprendras, tu sauras pourquoi il ne t'a pas compris» (Amadou Hampâté Bâ).

La maladie dans ces régions: «Cour des miracles d'un service de pédiatrie: des rougeoleux toussant leur insuffisance respiratoire, des tétaniques de deux ans tordus dans des rictus, les peaux pelées des kwashiorkors, le béri-béri et toute une kyrielle de parasitoses». Maurice King, éminent professeur de médecine en Afrique orientale il y a un demi-siècle, m'avait (J. M.) marqué: «Le facteur principal de la mauvaise santé dans les pays chauds n'est pas les maladies tropicales mais la pauvreté».

\section{«Ouvrage attachant, varié, bien écrit - notre confrère a une vraie plume.»}

Un certain nombre d'entre nous ont vécu des parties de leur carrière sur d'autres continents aux cadres de référence différents; voulant contribuer à «rendre le monde meilleur», lutter contre des situations inacceptables. Ils retrouveront au fil des pages l'une ou l'autre expérience, découverte, soulagement ou satisfaction, vicissitude ou désillusion, vécus dans ces engagements professionnels et humains; ils se souviendront de tel état d'esprit, questionnement ou rêverie. Mais il n'est pas besoin d'avoir été coopérant pour trouver à cette lecture intérêt et plaisir. Ouvrage attachant, varié, bien écrit - notre confrère a une vraie plume.

J'ai trouvé interpellants - et substantiels - les derniers chapitres, «Retour sur soi» et «La route, et après». Extraits: «C'est un livre qui réunit des souvenirs. Au départ, ce sont des faits. Avec le temps, on oublie leur réalité, on prend de la distance, on ne garde que ce qui a laissé une cicatrice dans la tête. Chaque jour qui passe, l'imaginaire grignote le réel, le laissant un peu plus disloqué. Ce faisant, le souvenir gagne en beauté». Et, en rapport avec l'interrogation évoquée plus haut: «Vous auriez voulu en savoir plus sur ce toubib. Il n'y a rien. Les mots ont été écrits pour nous confondre. Ce qui précède n'a pas plus de sens qu'un alignement de menhirs sur une lande bretonne». Well...!? Pourtant (note de J. M.) personne ne doute qu'un alignement de menhirs est porteur de sens, de beaucoup de sens. 